PROCEEDINGS OF THE

AMERICAN MATHEMATICAL SOCIETY

Volume 134, Number 9, September 2006, Pages 2667-2675

S 0002-9939(06)08290-6

Article electronically published on March 23, 2006

\title{
SCALED-OSCILLATION AND REGULARITY
}

\author{
ZOLTÁN M. BALOGH AND MARIANNA CSÖRNYEI \\ (Communicated by Juha M. Heinonen)
}

\begin{abstract}
We give sufficient conditions for Sobolev and Lipschitz functions in terms of their lower scaled-oscillation. The sharpness of these conditions is shown by examples. Our examples also show that a Stepanov-type differentiability theorem does not hold under the boundedness assumption of the lower scaled-oscillation.
\end{abstract}

\section{Introduction ANd StATEMEnt of RESUlts}

Let $(X, d, \mu)$ be a metric measure space and $f: X \rightarrow \mathbb{R}$ a continuous function. For $x \in X$ define the upper and lower scaled oscillation functions of $f$ as

$$
L_{f}(x)=\limsup _{r \rightarrow 0} \frac{\sup _{d(x, y) \leq r}|f(y)-f(x)|}{r}
$$

and

$$
l_{f}(x)=\liminf _{r \rightarrow 0} \frac{\sup _{d(x, y) \leq r}|f(y)-f(x)|}{r} .
$$

These functions were recently used by Keith Ke1, Ke2 to give sufficient conditions on $X$ under which the Cheeger-Rademacher theorem of differentiability of Lipschitz functions [C] holds.

Let us recall that if $f$ is a Lipschitz function, then there exists a constant $L>0$ such that $l_{f} \leq L_{f} \leq L$. If $X=\Omega \subset \mathbb{R}^{n}$ we can apply the classical Rademacher differentiability theorem according to which any Lipschitz function $f$ is differentiable for $\mathcal{L}^{n}$ a.e. $x \in \Omega$, and at the points of differentiability we have the equality

$$
L_{f}(x)=l_{f}(x)=|\nabla f(x)| .
$$

A generalization of Rademacher's theorem is Stepanov's theorem, stating that the previous conclusion also holds under the weaker assumption that $L_{f}(x)<\infty$ for $\mathcal{L}^{n}$ a.e. $x \in \Omega$. A generalization of Stepanov's theorem in the general setting of metric measures spaces was recently obtained in BRZ.

It is a natural question to study whether this differentiability result holds true if we replace $L_{f}$ by $l_{f}$; namely under the condition $l_{f}(x)<\infty$ for $\mathcal{L}^{n}$ a.e. $x \in \Omega$. In this paper we show that this is not the case, however the Sobolev and Lipschitz functions can be described in terms of some stronger conditions on the lower scaledoscillation function $l_{f}$.

This research was motivated by results of Heinonen and Koskela [HK], Balogh and Koskela [BK] and Kallunki and Koskela [KK1, where quasiconformal mappings

Received by the editors May 27, 2004 and, in revised form, April 3, 2005.

2000 Mathematics Subject Classification. Primary 26B35; Secondary 26B05. 
were characterized by certain similar liminf conditions. More recently Kallunki and Koskela [KK2], Ka] studied related questions for the more general class of mappings of finite distortion. Furthermore, in [MRSY] Martio, Ryazanov, Srebro and Yabukov studied general properties of space mappings of finite length distortion which are mappings for which the two functions $L_{f}$ and $l_{f}$ are finite and comparable almost everywhere.

It turns out that it is possible to give sufficient conditions on the lower scaledoscillation function $l_{f}$ for $f$ to belong to the Sobolev or Lipschitz class. In $\mathbb{R}^{n}$ it is in fact enough to assume that $l_{f}(x)<\infty$ outside an exceptional set $E$ of $\sigma$-finite $(n-1)$-dimensional Hausdorff measure, combined with the condition that $l_{f}$ belongs to an appropriate $L^{p}$ class. In more general Ahlfors regular metric spaces stronger assumptions on the size of the exceptional sets are needed. These results appear to be the right analogues of the corresponding results for quasiconformal mappings $[\mathrm{HK}],[\mathrm{BK}]$, KK1] and are related to removability results for Sobolev spaces [Ko], KST.

The starting point of our investigation is the following variant of a theorem of Banach.

Lemma 1.1. Let $f:[0,1] \rightarrow \mathbb{R}$ be a continuous function such that $l_{f}(x)<\infty$ for $x \in[0,1] \backslash E$, where the set $E$ is at most countable. Then $f$ is differentiable on a set of positive Lebesgue measure.

Assume also that $l_{f} \in L^{1}([0,1])$. Then $f$ is an absolutely continuous (AC) function. In particular $f$ is differentiable for $\mathcal{L}^{1}$ a.e. $x \in[0,1]$ and

$$
\left|f^{\prime}(x)\right|=l_{f}(x) \text { for } \mathcal{L}^{1} \quad \text { a.e. } x \in[0,1] .
$$

We use the above statement to prove the following higher-dimensional result.

Theorem 1.2. Let $\Omega \subseteq \mathbb{R}^{n}$ be a domain and $f: \Omega \rightarrow \mathbb{R}$ a continuous function. Assume that $l_{f}(x)<\infty$ for $x \in \Omega \backslash E$, where the exceptional set $E$ has $\sigma$-finite $(n-1)$-dimensional Hausdorff measure. Assume also that $l_{f} \in L_{l o c}^{p}(\Omega)$ for some $1 \leq p \leq \infty$. Then $f \in W_{\text {loc }}^{1, p}(\Omega)$. If, in addition $p>n$, we have

$$
l_{f}(x)=L_{f}(x)=|\nabla f(x)| \text { for } \mathcal{L}^{n} \text { a.e. } x \in \Omega .
$$

Let us observe that the above statement complements a result of Koskela $\mathrm{Ko}$ on removable sets for Sobolev spaces $W^{1, p}$. To see this recall that $E \subset \Omega$ is removable for the Sobolev space $W^{1, p}(\Omega)$ if $W^{1, p}(\Omega)=W^{1, p}(\Omega \backslash E)$ as sets. According to Ko, if $1<p \leq n$, then $E \subset \mathbb{R}^{n-1}$ is removable for $W^{1, p}$ if $E$ is a $p$-porous set. One can see that Theorem 1.2 implies the classical result that any $(n-1)$-rectifiable set $E \subset \Omega$ is removable for continuous Sobolev functions.

Theorem 1.2 is quite sharp. The following example shows that the integrability condition $l_{f} \in L^{1}$ is crucial. Without this condition, even under the strong pointwise assumption that $l_{f}(x)<\infty$ for all values of $x$, we cannot in general conclude the a.e. differentiability.

Theorem 1.3. There exist a set $A \subset[0,1]$ of positive Lebesgue measure and a continuous function $f:[0,1] \rightarrow \mathbb{R}$ such that $l_{f}(x)<\infty$ for all $x \in[0,1], l_{f} \in L^{s}[0,1]$ for all $s<1$ and $f$ is not differentiable at any point of $A$.

Note that in view of Lemma 1.1 the set $A$ in the above statement cannot be of full measure. 
Let us also mention here that if the exceptional set $E$ is empty, then Theorem 1.2 in the case $p=\infty$ becomes an exercise (solved, for example, by a covering argument).

It is a natural question to ask how large the set $E$ can be and still have the theorem remain true. The following example shows that the condition that $E$ has $\sigma$-finite $(n-1)$-dimensional Hausdorff measure cannot be replaced by asking $E$ to be of Lebesgue measure zero as in the case of Stepanov's theorem.

Theorem 1.4. There exists a nowhere differentiable, continuous function $f$ : $[0,1] \rightarrow \mathbb{R}$ such that $l_{f}(x)=0$ for $\mathcal{L}^{1}$ a.e. $x \in[0,1]$.

To formulate corresponding results in the general metric space setting we recall some basic notions from analysis in metric spaces. We refer to the recent book of Heinonen $[\mathrm{H}]$ for more details on the subject. We consider a metric measure space $(X, d, \mu)$ where $\mu$ is an Ahlfors $Q$-regular Borel measure for some $Q>0$, i.e. there exists a constant $C \geq 1$ such that for $x \in X$ and $r<\operatorname{diam} X$ we have

$$
\frac{1}{C} \cdot r^{Q} \leq \mu(B(x, r)) \leq C \cdot r^{Q}
$$

where $B(x, r)$ stands for the metric ball with center $x$ and radius $r$. For a family $\Gamma$ of rectifiable curves in $X$ and $p>0$ one defines the $p$-modulus $\operatorname{Mod}_{p}(\Gamma)$ as

$$
\operatorname{Mod}_{p}(\Gamma)=\inf \int_{X} \varrho^{p} d \mu,
$$

where the infimum is taken over the family of all Borel functions $\varrho: X \rightarrow[0, \infty]$ for which $\int_{\gamma} \varrho d s \geq 1$ for each $\gamma \in \Gamma$. A possible generalization of the notion of the "gradient" from $\mathbb{R}^{n}$ to the general metric setting is the following concept. We say that a Borel function $g: X \rightarrow[0, \infty]$ is an upper gradient of the function $u: X \rightarrow \mathbb{R}$ if

$$
|u(x)-u(y)| \leq \int_{\gamma} g d s,
$$

for each rectifiable curve $\gamma$ joining $x$ and $y$ in $X$. The function $g$ is called a $p$-weak upper gradient of $u$ if (1.2) holds except for a family of curves with vanishing $p$ modulus. A generalization of Sobolev spaces to the metric setting is the Newtonian space $N^{1, p}(X)(1 \leq p \leq \infty)$ due to Shanmungalingan [Sh]. The space $N^{1, p}(X)$ consists of those $L^{p}(X)$ functions $u: X \rightarrow \mathbb{R}$ which have a $p$-weak upper gradient in $L^{p}(X)$.

Theorem 1.5. Let $(X, d, \mu)$ be a $Q$-regular metric-measure space and $f: X \rightarrow \mathbb{R}$ a continuous function. Assume that there exists a set $E \subset X$ and an exponent $1 \leq p<Q$ such that $l_{f} \in L^{p}(X)$ and $l_{f}(x)<\infty$ for $x \in X \backslash E$, where $\mathcal{H}^{Q-p}(E)=0$. Then it follows that $f \in N^{1, p}(X)$.

In the above statement $\mathcal{H}^{Q-p}$ stands for the $(Q-p)$-dimensional Hausdorff measure with respect to the metric $d$ in $X$. Let us note that the statement in the general setting is substantially weaker than the result in $\mathbb{R}^{n}$. This is due to the fact that the Euclidean space has a fibration by lines parallel to the coordinate axes.

The paper has two sections: in the first one we give the proof of Lemma 1.1 and Theorems 1.2 and 1.5, and in the second section we present the constructions of the examples stated in Theorems 1.3 and 1.4 . 


\section{Proof of Theorems 1.2 and 1.5}

Proof of Lemma 1.1. By theorems of Banach (see e.g. Theorem IX.7.9 and IX.7.7 in $[\mathrm{S}]$ ), if a continuous function $f$ fulfils Luzin condition $(\mathrm{N})$ on an interval, then it is differentiable at the points of a set of positive measure. Furthermore, in order that $f$ be absolutely continuous on $[0,1]$, it is necessary and sufficient that $f$ simultaneously fulfils the Luzin condition $(\mathrm{N})$ and the condition

$$
\int_{P} f^{\prime}(x) d x<\infty
$$

where $P$ denotes the set of points at which $f$ has a finite non-negative derivative. (By definition, a function $f$ fulfils the Luzin condition (N) if $f(H)$ has measure zero for every Lebesgue null set $H \subset[0,1]$.)

First we show that $f$ fulfils condition $(\mathrm{N})$ : let $E^{\prime}$ denote the set of points at which $l_{f}$ is finite. Since $[0,1] \backslash E^{\prime}$ is at most countable, it is enough to check that $f$ fulfils $(\mathrm{N})$ on $E^{\prime}$. But $f$ fulfils condition (D) at each point of $E^{\prime}$ (by definition, $f$ fulfils condition (D) at $x$ if there is an $N$ such that the set $\{y:|f(y)-f(x)| \leq N|y-x|\}$ has positive lower density at $x$; for $x \in E^{\prime}$ this is satisfied with $N=2 l_{f}(x)$ ). By Theorem IX.9.7 in $\left[\underline{S}\right.$, if a function $f$ fulfils condition (D) at each point of a set $E^{\prime}$, then it fulfils condition $(\mathrm{N})$ on $E^{\prime}$. This implies that $f$ is differentiable on a set of positive measure.

It is also immediate to see that if $f$ is differentiable at a point $x$, then $\left|f^{\prime}(x)\right|=$ $l_{f}(x)$. Therefore if $l_{f} \in L^{1}([0,1])$, then (2.1) is satisfied and $f$ is absolutely continuous.

Let us observe that in the above lemma the continuity assumption on $f$ was essential. Indeed, just consider a piecewise constant non-continuous function $f$ such that $l_{f}(x)=0$ for $x \in[0,1] \backslash\{1 / 2\}$.

Proof of Theorem 1.2. According to Theorem 2.1.4 on p. 44 in [Z] it is enough to check that $f$ has the (ACL) property, i.e. it is absolutely continuous on almost every line parallel to the coordinate axes, and that the partial derivatives satisfy

$$
\frac{\partial f}{\partial x_{i}} \in L_{\mathrm{loc}}^{p}(\Omega) \text { for } i=1, \ldots, n .
$$

We shall only consider lines parallel to the $x_{n}$-axis that are of the form $\left\{x+t e_{n}\right.$ : $t \in \mathbb{R}\}$.

Let $\Omega^{\prime} \subset \Omega$ be a subdomain such that

$$
d=\operatorname{dist}\left(\Omega^{\prime}, \partial \Omega\right)>0 .
$$

Without loss of generality assume that $\Omega^{\prime}$ is the unit cube in $\mathbb{R}^{n} ; \Omega^{\prime}=\Omega_{n-1}^{\prime} \times[0,1]$, where $\Omega_{n-1}^{\prime}$ is the unit cube in $\mathbb{R}^{n-1}$. It is easy to see that for $\mathcal{L}^{n-1}$ a.e. $x \in \Omega_{n-1}^{\prime}$ the one-variable function $g_{x}: t \rightarrow f\left(x+t e_{n}\right)$ is absolutely continuous. Indeed, by Fubini's theorem $l_{f} \in L^{p}\left(\left[x, x+e_{n}\right]\right)$ for a.e. $x \in \Omega_{n-1}^{\prime}$ and $\left[x, x+e_{n}\right] \cap E$ is at most countable (see e.g. $[\mathrm{V}]$, p. 105). Since $l_{g_{x}}(t) \leq l_{f}\left(x+t e_{n}\right)$, by applying Lemma 1.1 it follows that $g_{x}$ is (AC) for a.e. $x \in \Omega_{n-1}^{\prime}$.

Using the fact that for a.e. $x \in \Omega_{n-1}^{\prime}$ and $\mathcal{L}^{1}$ a.e. $t \in[0,1]$ we have

$$
\left|\frac{\partial f}{\partial x_{n}}\left(x+t e_{n}\right)\right|=\left|g_{x}^{\prime}(t)\right|=l_{g_{x}}(t) \leq l_{f}\left(x+t e_{n}\right),
$$


and by applying Fubini's theorem it follows that

$$
\int_{\Omega}\left|\frac{\partial f}{\partial x_{n}}(x)\right|^{p} d x \leq \int_{\Omega} l_{f}^{p}(x) d x
$$

finishing the proof of the first statement. The second statement follows from the fact that for $p>n$ functions in $W^{1, p}$ are differentiable almost everywhere.

Proof of Theorem 1.5. It is a well-known fact (see $\mathrm{H}$, p. 55) that if $f$ is a Lipschitz function, then $l_{f}$ is an upper gradient for $f$. Our proof generalizes this as we check that $l_{f}$ is a weak upper gradient of $f$.

Let $\Gamma_{E}$ denote the family of all rectifiable curves that meet $E$. It is enough to prove that if $\mathcal{H}^{Q-p}(E)=0$, then $\operatorname{Mod}_{p}\left(\Gamma_{E}\right)=0$. Indeed, we can apply Lemma 1.1 for any curve $\gamma \notin \Gamma_{E}$ for which $l_{f} \circ \gamma \in L^{p}$, and, by definition, $l_{f} \in L^{p}(X)$ implies that $l_{f} \circ \gamma$ is in $L^{p}$ for all rectifiable curves $\gamma$ except for a family of curves of vanishing $p$-modulus. Let $\Gamma_{E}^{k}$ denote those curves in $\Gamma_{E}$ whose length is at least $1 / k$. We prove that $\operatorname{Mod}_{p}\left(\Gamma_{E}^{k}\right)=0$.

Let $\varepsilon>0$ be arbitrary, and let $\left\{B_{i}\right\}_{i \in I}$ be a countable system of balls $B_{i}=$ $B\left(x_{i}, r_{i}\right)$ whose union covers $E$ and for which $\sum_{i} r_{i}^{Q-p}<\varepsilon$ and $r_{i}<1 / k$ for each $i$. We choose a subset of indices $J \subset I$ such that $\bigcup_{j \in J} 5 B_{j}$ covers $E$ and the balls $B_{j}, j \in J$, are pairwise disjoint. Let

$$
\varrho=\sum_{j \in J} \frac{1}{r_{j}} \cdot \chi_{6 B_{j}} .
$$

Notice that, if a curve $\gamma$ meets $E$, then it meets at least one of the balls $5 B_{j}$, and if $\gamma$ has length at least $1 / k \geq r_{j}$, then it meets $6 B_{j}$ in a curve of length at least $r_{j}$. Hence for any $\gamma \in \Gamma_{E}^{k}: \int_{\gamma} \varrho d s \geq \max _{j} \frac{1}{r_{j}} \mathcal{H}^{1}\left(\gamma \cap 6 B_{j}\right) \geq 1$, and therefore

$$
\operatorname{Mod}_{p}\left(\Gamma_{E}^{k}\right) \leq \int_{X} \varrho^{p} d \mu=\int_{X}\left(\sum_{j \in J} \frac{1}{r_{j}} \cdot \chi_{6 B_{j}}\right)^{p} d \mu \leq K \int_{X}\left(\sum_{j \in J} \frac{1}{r_{j}} \cdot \chi_{B_{j}}\right)^{p} d \mu .
$$

The last inequality holds for any doubling measure space with a constant $K$ that depends only on the measure $\mu$ (see e.g. $[\mathrm{H}]$, p. 13).

Since the balls $B_{j}$ are disjoint, by the $Q$-regularity of $\mu$ the right-hand side is estimated as

$$
K \cdot \sum_{j \in J} \frac{1}{r_{j}^{p}} \cdot \mu\left(B_{j}\right) \leq K C \cdot \sum_{j \in J} r_{j}^{Q-p}<K C / \varepsilon .
$$

This finishes the proof.

If the exceptional set $E$ is at most countable, the result of Theorem 1.5 remains true for $p \geq Q$. If in addition the space $(X, d, \mu)$ satisfies a $p$-Poincaré inequality, then we can conclude (cf. [Sh, [BRZ]) that $f$ is $(1-p / Q)$-Hölder continuous and $\mu$ a.e. differentiable in the sense of Cheeger.

\section{Proofs of Theorems 1.3 And 1.4}

The construction of the examples stated in Theorems 1.3 and 1.4 is based on an iterative argument whose building-block is the following simple geometric observa- 
tion: For a given rectangle $R=\left[x_{1}, x_{2}\right] \times\left[y_{1}, y_{2}\right] \subset[0,1]^{2}$ and $0<\varepsilon<\frac{x_{2}-x_{1}}{y_{2}-y_{1}}<1 / 2$ let

$$
\begin{aligned}
& x_{3}=x_{2}-\varepsilon\left(y_{2}-y_{1}\right) \\
& y_{3}=y_{1}+\varepsilon\left(x_{3}-x_{1}\right) / 2 \\
& y_{4}=y_{2}-\varepsilon\left(x_{3}-x_{1}\right) / 2 \\
& y_{5}=(1 / 2-\varepsilon / 2) y_{1}+(1 / 2+q \varepsilon / 2) y_{2} \\
& y_{6}=(1 / 2+\varepsilon / 2) y_{1}+(1 / 2-\varepsilon / 2) y_{2} .
\end{aligned}
$$

Let $T_{1, R, \varepsilon}, T_{2, R, \varepsilon}, T_{3, R, \varepsilon}$ and $T_{4, R, \varepsilon}$ denote the triangles with vertices

$$
\begin{aligned}
& \left(x_{1}, y_{1}\right),\left(x_{3}, y_{1}\right),\left(\frac{x_{1}+x_{3}}{2}, y_{3}\right) ; \\
& \left(x_{3}, y_{1}\right),\left(\frac{x_{3}+x_{2}}{2}, y_{5}\right),\left(x_{2}, y_{2}\right) ; \\
& \left(x_{1}, y_{2}\right),\left(x_{3}, y_{2}\right),\left(\frac{x_{1}+x_{3}}{2}, y_{4}\right) ; \\
& \left(x_{3}, y_{2}\right),\left(\frac{x_{3}+x_{2}}{2}, y_{6}\right),\left(x_{2}, y_{1}\right)
\end{aligned}
$$

respectively. An easy computation shows that for every function $f:[0,1] \rightarrow \mathbb{R}$ for which the graph of $\left.f\right|_{\left[x_{1}, x_{2}\right]}$ lies either in $T_{1, R, \varepsilon} \cup T_{2, R, \varepsilon}$ or in $T_{3, R, \varepsilon} \cup T_{4, R, \varepsilon}$ there holds

(i) $\frac{\sup _{|y-x| \leq r}|f(y)-f(x)|}{r} \leq \varepsilon$ for $x \in\left(x_{1}, x_{3}\right)$ and $r=\min \left(x_{3}-x, x-x_{1}\right)$;

(ii) $\frac{\sup _{0<y-x \leq r}|f(y)-f(x)|}{r} \leq \varepsilon$ for $x=x_{1}$ and for every $r \leq x_{3}-x_{1}$;

(iii) $\frac{\sup _{-r \leq y-x<0}|f(y)-f(x)|}{r} \leq \varepsilon$ for $x=x_{3}$ and for every $r \leq x_{3}-x_{1}$;

(iv) $\frac{|f(y)-f(x)|}{|y-x|} \geq \frac{y_{2}-y_{1}}{x_{2}-x_{3}}=1 / \varepsilon$ for $x=x_{3}$ and for every $y \in\left[x_{3}, x_{2}\right]$;

(v) $\frac{|f(y)-f(x)|}{|y-x|}>2-\varepsilon / 2$ for $x=x_{2}$ and for every $y \in\left[x_{1}, x_{3}\right]$;

(vi) $\frac{|f(y)-f(x)|}{|y-x|} \geq \frac{y_{2}-y_{5}}{\left(x_{2}-x_{3}\right) / 2}>\frac{1 / 2-\varepsilon / 2}{\varepsilon / 2}>\frac{1}{2 \varepsilon}$ for $x=x_{2}$ and for every $y \in\left[x_{3}, x_{2}\right]$.

Therefore, if for a given continuous function $f:[0,1] \rightarrow \mathbb{R}$ and for a given point $x \in[0,1]$ one can find arbitrarily small values $\varepsilon>0$ and arbitrarily small rectangles $R=\left[x_{1}, x_{2}\right] \times\left[y_{1}, y_{2}\right]$ with $\varepsilon<\frac{x_{2}-x_{1}}{y_{2}-y_{1}}<1 / 2$, such that $x \in\left(x_{1}, x_{2}\right)$ and the graph of $\left.f\right|_{\left[x_{1}, x_{2}\right]}$ lies either in $T_{1, R, \varepsilon} \cup T_{2, R, \varepsilon}$ or in $T_{3, R, \varepsilon} \cup T_{4, R, \varepsilon}$, then $f$ is not differentiable at $x$. Moreover, from (i)-(vi) we can conclude the following:

Claim. Let $f:[0,1] \rightarrow \mathbb{R}$ be a continuous function and $x \in[0,1]$.

(a) If there exist arbitrarily small values $\varepsilon>0$ and arbitrarily small rectangles $R=\left[x_{1}, x_{2}\right] \times\left[y_{1}, y_{2}\right]$ with $\varepsilon<\frac{x_{2}-x_{1}}{y_{2}-y_{1}}<1 / 2$, such that $x \in\left(x_{1}, x_{3}\right)$ and such that the graph of $\left.f\right|_{\left[x_{1}, x_{2}\right]}$ lies either in $T_{1, R, \varepsilon} \cup T_{2, R, \varepsilon}$ or in $T_{3, R, \varepsilon} \cup T_{4, R, \varepsilon}$, then $l_{f}(x)=0$ and $L_{f}(x) \geq 2$.

(b) If there exist arbitrarily small values $\varepsilon>0$ and arbitrarily small rectangles $R=\left[x_{1}, x_{2}\right] \times\left[y_{1}, y_{2}\right]$ with $\varepsilon<\frac{x_{2}-x_{1}}{y_{2}-y_{1}}<1 / 2$, such that $x \in\left[x_{3}, x_{2}\right]$ and such that the graph of $\left.f\right|_{\left[x_{1}, x_{2}\right]}$ lies either in $T_{1, R, \varepsilon} \cup T_{2, R, \varepsilon}$ or in $T_{3, R, \varepsilon} \cup T_{4, R, \varepsilon}$, then $L_{f}(x)=\infty$.

(c) If there exist an $\varepsilon>0$ and a rectangle $R=\left[x_{1}, x_{2}\right] \times\left[y_{1}, y_{2}\right]$ with $\varepsilon<$ $\frac{x_{2}-x_{1}}{y_{2}-y_{1}}<1 / 2$, such that the graph of $\left.f\right|_{\left[x_{1}, x_{2}\right]}$ lies either in $T_{1, R, \varepsilon} \cup T_{2, R, \varepsilon}$ or in $T_{3, R, \varepsilon} \cup T_{4, R, \varepsilon}$, then

(c1) $\lim \sup _{y-x_{1} \rightarrow 0^{+}}\left|\frac{f(y)-f\left(x_{1}\right)}{y-x_{1}}\right| \leq \varepsilon$;

(c2) $\lim \sup _{y-x_{3} \rightarrow 0^{-}}\left|\frac{f(y)-f\left(x_{3}\right)}{y-x_{3}}\right| \leq \varepsilon<1<1 / \varepsilon$ $\leq \limsup _{y-x_{3} \rightarrow 0^{+}}\left|\frac{f(y)-f\left(x_{3}\right)}{y-x_{3}}\right|$

(c3) $\lim \sup _{y-x_{2} \rightarrow 0^{-}}\left|\frac{f(y)-f\left(x_{2}\right)}{y-x_{2}}\right|>1 / 2 \varepsilon$. 
Indeed, (a) follows from (i) and (v), (b) follows from (iv), (c1) follows from (ii), (c2) follows from (iii) and (iv), and finally (c3) follows from (vi).

Our strategy of constructing a function $f$ as stated in Theorems 1.3, 1.4 is as follows. By induction for any $m \in \mathbb{N}$ and sequence of integers $\underline{n}=\left(n_{1}, n_{2}, \ldots, n_{m}\right)$ we define triangles $T_{\underline{n}} \subset[0,1]^{2}$ with vertices $\left(a_{\underline{n}}, a_{\underline{n}}^{*}\right),\left(b_{\underline{n}}, b_{\underline{n}}^{*}\right),\left(c_{\underline{n}}, c_{\underline{n}}^{*}\right)$, where $a_{\underline{n}}<$ $c_{\underline{n}}<b_{\underline{n}}$. We will choose the triangles $T_{\underline{n}}$ in such a way that for every $\underline{n}$ and integer $k$ :

(I) $T_{\underline{n}} \supset T_{\underline{n}, k}$;

(II) $\operatorname{diam} T_{\underline{n}} \leq 1 / m$;

(III) $\cdots \leq a_{\underline{n}, k}<b_{\underline{n}, k} \leq a_{\underline{n}, k+1}<b_{\underline{n}, k+1} \leq \cdots$;

(IV) $\lim _{k \rightarrow-\infty} T_{\underline{n}, k}=\left(a_{\underline{n}}, a_{\underline{n}}^{*}\right)$ and $\lim _{k \rightarrow \infty} T_{\underline{n}, k}=\left(b_{\underline{n}}, b_{\underline{n}}^{*}\right)$;

(V) if $b_{\underline{n}, k}=a_{\underline{n}, k+1}$, then $b_{\underline{n}, k}^{*}=a_{\underline{n}, k+1}^{*}$.

To a sequence of triangles as above we associate a function defined as follows. Set

$$
f\left(a_{\underline{n}, k}\right)=a_{\underline{n}, k}^{*}, f\left(b_{\underline{n}, k}\right)=b_{\underline{n}, k}^{*}
$$

for each $k$, and extend $f$ to the intervals $\left[b_{\underline{n}, k}, a_{\underline{n}, k+1}\right]$ linearly. On the intervals $\left[a_{\underline{n}, k}, b_{\underline{n}, k}\right]$ we require

$$
\text { graph }\left.f\right|_{\left[a_{\underline{n}, k}, b_{\underline{n}, k}\right]} \subset T_{\underline{n}, k} .
$$

Since in the $m$ th step by (II) diam $T_{\underline{n}} \leq 1 / m \rightarrow 0$ as $m \rightarrow \infty$, and since by (I) $T_{\underline{n}} \supset T_{\underline{n}, k}$, the function $f$ is well defined on the whole interval $[0,1]$. One checks easily that it is also continuous.

Proof of Theorem 1.4. The proof is based on a special choice of a sequence of triangles that are related to the rectangles described at the beginning of this section.

Let $T_{\emptyset}$ be the triangle with vertices $(0,0),(1 / 2,0),(1,1)$. If for some $m \in \mathbb{N}$ and $\underline{n}=\left(n_{1}, n_{2}, \ldots, n_{m}\right)$ the triangle $T_{\underline{n}}$ has been defined, then we choose points $\left(t_{k}, u_{k}\right)$ for every integer $k$ such that

(A) $0<3 m\left(t_{k+1}-t_{k}\right)=\left|u_{k+1}-u_{k}\right| \leq 1 / 2 m$;

(B) $\left[t_{k}, t_{k+1}\right] \times\left[\min \left(u_{k}, u_{k+1}\right), \max \left(u_{k}, u_{k+1}\right)\right] \subset T_{\underline{n}}$;

(C) $\lim _{k \rightarrow-\infty}\left(t_{k}, u_{k}\right)=\left(a_{\underline{n}}, a_{\underline{n}}^{*}\right)$ and $\lim _{k \rightarrow \infty}\left(t_{k}, u_{k}\right)=\left(b_{\underline{n}}, b_{\underline{n}}^{*}\right)$.

Let $R_{k}=\left[t_{k}, t_{k+1}\right] \times\left[\min \left(u_{k}, u_{k+1}\right), \max \left(u_{k}, u_{k+1}\right)\right]$ and $\varepsilon=\frac{1}{3\left(m^{3}+1\right)}$. Then

$$
0<\varepsilon<\frac{t_{k+1}-t_{k}}{\left|u_{k+1}-u_{k}\right|}=\frac{1}{3 m}<1 / 2 .
$$

We define $T_{\underline{n}, 2 k}=T_{1, R_{k}, \varepsilon}$ and $T_{\underline{n}, 2 k+1}=T_{2, R_{k}, \varepsilon}$ if $u_{k}<u_{k+1}$, and $T_{\underline{n}, 2 k}=T_{3, R_{k}, \varepsilon}$ and $T_{\underline{n}, 2 k+1}=T_{4, R_{k}, \varepsilon}$ if $u_{k}>u_{k+1}$. This choice of triangles satisfies (I)-(V) and hence it defines a continuous function $f$.

From part (a) of our Claim it follows that $l_{f}(x)=0$ at each point $x$ for which $(x, f(x))$ is covered by the interior of infinitely many triangles of the form $T_{\underline{n}, 2 k}$. On the other hand

$$
b_{\underline{n}, 2 k+1}-a_{\underline{n}, 2 k+1}=\varepsilon\left|u_{k+1}-u_{k}\right|=\frac{3 m\left(t_{k+1}-t_{k}\right)}{3 m^{3}+1}<\left(t_{k+1}-t_{k}\right) / m^{2},
$$

hence

$$
\mathcal{L}^{1}\left(\left\{x:(x, f(x)) \notin \bigcup_{\underline{n} \in \mathbb{Z}^{m}, k \in \mathbb{Z}} T_{\underline{n}, 2 k}\right\}\right)<1 / m^{2} .
$$

Therefore $l_{f}(x)=0$ for $\mathcal{L}^{1}$ a.e. $x$. 
From parts (a)-(c) of our Claim it also follows that $f$ is not differentiable anywhere. To see this notice that every point $x \in[0,1]$ is included in a nested sequence of infinitely many triangles for which one of the cases (a), (b) or (c) from our claim holds. In case (a) we have $l_{f}(x)=0<2 \leq L_{f}(x)$, in case (b) we know that $L_{f}(x)=\infty$, and in case (c) the absolute value of one of the two one-sided derivatives of $f$ at $x$ is zero and the other is infinite.

Proof of Theorem 1.3. We proceed similarly as in the proof of Theorem 1.4. First we define $T_{\emptyset}$, and if for some $\underline{n}$ the triangle $T_{\underline{n}}$ has been defined, then we choose $t_{k}, u_{k}$ that satisfy (A)-(C), and we define $R_{k}$ and $\varepsilon$ the same way as before. But then we choose the triangles $T_{\underline{n}, k}$ differently: let $T_{\underline{n}, k}=T_{1, R_{k}, \varepsilon}$ if $u_{k}<u_{k+1}$ and let $T_{\underline{n}, k}=T_{3, R_{k}, \varepsilon}$ if $u_{k}>u_{k+1}$. We claim that the function $f$ defined by this system of triangles satisfies Theorem 1.3.

Let $A$ be the set of those points which are covered by infinitely many triangles of our construction. Since

$b_{\underline{n}, k}-a_{\underline{n}, k}=\left(t_{k+1}-t_{k}\right)-\varepsilon\left|u_{k+1}-u_{k}\right|=\left(t_{k+1}-t_{k}\right)(1-3 m \varepsilon)>\left(t_{k+1}-t_{k}\right)\left(1-1 / m^{2}\right)$, the measure of $A$ is greater than $\prod_{m=1}^{\infty}\left(1-1 / m^{2}\right)>0$.

By (a) of our Claim we know that $l_{f}(x)=0$ at each point of $A$, and that $f$ is not differentiable at these points. We need to check that $l_{f}(x)<\infty$ at every $x \notin A$.

This is also immediate, since these points are either on a piece where $f$ is linear, or they are the endpoints of such a piece. At the endpoints on one side the function is linear and on the other side

$$
\begin{aligned}
& \text { for } x=a_{\underline{n}} \text { it follows from (c1) that } \limsup _{y-x \rightarrow 0^{+}}\left|\frac{f(y)-f(x)}{y-x}\right| \leq \varepsilon ; \\
& \text { for } x=b_{\underline{n}} \text { it follows from (c2) that } \limsup _{y-x \rightarrow 0^{-}}\left|\frac{f(y)-f(x)}{y-x}\right| \leq \varepsilon .
\end{aligned}
$$

By choosing a sequence $\varepsilon_{m}$ that goes to zero sufficiently fast and by choosing $\varepsilon=\varepsilon_{m}$ in the $m$ th step, the above construction gives a continuous function $f$ for which $l_{f} \in \bigcap_{s<1} L^{s}([0,1])$. We leave the details to the reader.

\section{ACKNOWLEDGEMENT}

We thank Pekka Koskela and Hans Martin Reimann for stimulating conversations and advice on the subject of this paper, and David Preiss for simplifying our arguments. We also thank the referee for carefully reading the paper and for her/his comments.

\section{REFERENCES}

[BK] Z. M. Balogh, P. Koskela, Quasiconformality, quasisymmetry, and removability in Loewner spaces, Duke Math. J. 101 (2000), no. 3, 554-577. MR1740689 (2001d:30029)

[BRZ] Z. M. Balogh, K. Rogovin, T. Zürcher, The Stepanov differentiability theorem in metric measure spaces., J. Geom. Anal. 14 (2004), no. 3, 405-422. MR.2077159 (2005d:28008)

[C] J. Cheeger, Differentiability of Lipschitz functions on metric measure spaces, Geom. Funct. Anal. 9 (1999), no. 3, 428-517. MR.1708448 (2000g:53043)

$[\mathrm{H}] \quad$ J. Heinonen, Lectures on Analysis on Metric Spaces, Universitext, Springer Verlag (2001). MR1800917 (2002c:30028)

[HK] J. M. Heinonen, P. Koskela, Definitions of quasiconformality, Invent. Math. 120 (1995), 61-79. MR.1323982 (96e:30051)

[Ka] S. Kallunki, Mappings of finite distortion: the metric definition, Ann. Acad. Sci. Fenn. Dissertationes 131 (2002). MR1928755 (2003g:30036)

[Ke1] S. Keith, A differentiable structure for metric measure spaces, Advances in Math. 183 (2004), no. 2, 271-315. MR2041901(2005g:46070) 
[Ke2] S. Keith, Measurable differentiable structures and the Poincaré inequality, Indiana Univ. Math. J. 53 (2004), no. 4, 1127-1150. MR2095451 (2005g:53068)

[KM] J. Kinnunen, O. Martio, The Sobolev capacity on metric spaces. Ann. Acad. Sci. Fenn. Math. 21 (1996), no. 2, 367-382. MR1404091 (98c:46063)

[KK1] S. Kallunki, P. Koskela, Exceptional sets for the definitions of quasiconformality, Amer. J. Math. 122 (2000), 735-743. MR.1771571 (2001h:37095)

[KK2] S. Kallunki, P. Koskela, Metric definition of $\mu$-homeomorphisms, Mich. Math. J. 51 (2003), 141-152. MR:1960925 (2004h:30024)

[Ko] P. Koskela, Removable sets for Sobolev spaces, Ark. Mat. 37 (1999), no. 2, 291-304. MR 1714767 (2001g:46077)

[KST] P. Koskela, N. Shanmugalingam, H. Tuominen, Removable sets for the Poincaré inequality on metric spaces. Indiana Univ. Math. J. 49 (2000), no. 1, 333-352. MR.1777027 (2001g:46076)

[MRSY] O. Martio, V. Ryazanov, U. Srebro, E. Yabukov, Mappings of finite length distortion, to appear in J. d'Analyse Math.

[S] S. Saks, Theory of the Integral, Monografie Matematyczne, Warsaw, 1937.

[Sh] N. Shanmugalingam, Newtonian spaces: an extension of Sobolev spaces to metric spaces, Rev. Mat. Iberoamericana. 16 (2000), no. 2, 243-279. MR1809341 (2002b:46059)

[V] J. Väisälä, Lectures on n-dimensional Quasiconformal Mappings, Lecture Notes in Math. 229, Springer-Verlag (1971). MR0454009 (56:12260)

[Z] W. P. Ziemer, Weakly differentiable functions, Graduate Texts in Math. 120 Springer Verlag (1989). MR1014685 (91e:46046)

Mathematisches Institut, Universität Bern, CH-3012 Sidlerstrasse 5, Bern, SwitzerLAND

E-mail address: zoltan.balogh@math-stat.unibe.ch

Department of Mathematics, University College London, Gower Street, London WC1E 6BT, United KingDom

E-mail address: mari@math.ucl.ac.uk 